\title{
In-line hologram segmentation for volumetric samples
}

\author{
László Orzó, ${ }^{1, *}$ Zoltán Göröcs, ${ }^{1}$ András Fehér, ${ }^{1}$ and Szabolcs Tökés ${ }^{1,2}$ \\ ${ }^{1}$ Cellular Sensory and Wave Computing Laboratory, Computer and Automation Research \\ Institute, Hungarian Academy of Sciences, \\ H-1111 Budapest, Hungary \\ ${ }^{2}$ Faculty of Information Technology, Pázmány Péter Catholic University, \\ H-1083 Budapest, Hungary \\ *Corresponding author: orzo@sztaki.hu
}

\begin{abstract}
We propose a fast, non-iterative method to segment an in-line hologram of a volumetric sample into in-line sub-holograms according to its constituent objects. In contrast to the phase retrieval or twin image elimination algorithms we do not aim or require to reconstruct the complex wave field of all the objects, which would be a more complex task, but only provide a good estimate about the contribution of the particular objects to the original hologram quickly. The introduced hologram segmentation algorithm exploits the special inner structure of the in-line holograms and applies only the estimated supports and reconstruction distances of the corresponding objects as parameters. The performance of the proposed method is demonstrated and analyzed experimentally both on synthetic and measured holograms. We discussed how the proposed algorithm can be efficiently applied for object reconstruction and phase retrieval tasks. (c) 2012 Optical Society of America
\end{abstract} OCIS codes: 090.1995, 100.2000, 100.3010, 180.3170.

\section{Introduction}

From a recorded hologram it is possible to reconstruct its constituent objects with high resolution even if they are in different distances from the hologram [1]. This feature can be utilized in Digital Holographic Microscopy (DHM), where the resolution defined depth of focus constraint of conventional microscopy can be bypassed this way. Consequently, the observable volume considerably increases [2,3]. Applying a DHM system, we can implement a holographic flow cytometer (Fig 1(a)), that records the hologram of freely moving objects within a flow through sample chamber by a high resolution area scan (CCD or CMOS) sensor. 
These emerging objects, even if their diffractions overlap in the recorded hologram (Fig 1(b)), can be digitally reconstructed later, together with their 3D positions [4 6]. Contrary to other existing techniques [4], we use fast, non-iterative methods to find, segment and reconstruct the objects within the sample volume at a high resolution $(\lesssim 1 \mu \mathrm{m})$, with their actual 3D positions. In this way, an extremely efficient fluid inspection or water monitoring system can be constructed [7,8].

From a recorded hologram it is possible to recover even the whole complex wave field of a given object, namely not only its amplitude but also its phase distribution. This reconstruction is straightforward if an off-axis setup is applied [9, 10]. From the reconstructed complex wave field of a given object, the apparent thickness, shape and their changes become measureable with sub-wavelength accuracy [11,12. However, in the case of off-axis holograms the necessary spatial separation of the so-called object term from the zero order and twin image terms decrease the effective aperture of the hologram [13]. Due to this decay of the effective aperture, the trade-off between the achievable lateral resolution and field of view becomes more pronounced. Therefore, using an off-axis setup the size of the observable volume shrinks considerably. This is also the case, when the application of phase shifting interference microscopy 14, 15 is considered. Although phase shifting method would provide a simple, elegant way of complex wave field reconstruction, it requires several hologram recording steps that considerably reduce the achievable speed of volume inspection. Furthermore, it is hardly applicable, when moving, varying objects are to be measured.

Due to its simplicity and efficient utilization of sensor resolution the application of inline holographic setups are commonly preferred 16, 17]. Especially, the conventional Gabor holographic architecture is favored, where coherent illumination is applied in a more or less conventional digital microscope setup. In this case, the illuminating wave field serves also as the reference. This architecture provides a really simple measuring setup (Fig 1(a)) and applicable whenever the wave field of the objects can be considered only as a perturbation of the reference, that is, sparse, slightly scattering objects are to be recorded [16, 18. Using such an architecture, even lensless DHM systems become realizable [19, 20].

Constructing a special DHM setup we can avoid the limitations caused by the small depth of focus of the conventional microscopes and the observable volume becomes orders of magnitudes larger. Our device records in-line holograms of the objects within a large $\left(\approx 1 \mathrm{~mm}^{3}\right)$ sample volume. To ensure the lateral resolution of at least $1 \mu \mathrm{m}$ over the entire volume, slight, diffraction limited optical magnification (5x, using Olympus objectives) was applied besides a high resolution (9 megapixels, pixel size of $1.75 \mu \mathrm{m}$ ) CMOS sensor. Special fiber coupled lasers provide the illumination and guarantee the even spherical reference wave field in this device [7]. In contrast to other DHM approaches the objects spread all over a substantial volume and do not concentrate in a thin layer [11, 12, 20. Therefore, 
their diffractions can considerably overlap not only at the hologram recording but also at the reconstruction plane. The introduced hologram segmentation method can be applied efficiently to decrease or eliminate these distracting diffractions.

Using an in-line hologram the twin image overlaps the reconstructed object, which can considerably corrupt the quality of the reconstruction. If the object is far from the hologram plane the separation of the twin image from the object is straightforward [21] and sometimes it can be neglected [16]. However, when high resolution reconstruction is aimed, the objects have to be relatively close to the hologram recording plane to ensure the high effective numeric aperture. In this case, the twin image considerably overlaps with the reconstruction of the object and the twin image removal becomes a really challenging task [22, 23]. By the application of proper phase retrieval (twin image elimination) algorithms this type of bias of the object reconstruction can be decreased or eliminated [24]. Some phase retrieval algorithms iteratively use the spatial (i.e. finite support) and hologram amplitude constraints 25].

Although these algorithms, like the modified Gerchberg-Saxton [23], or its Fienup accelerated variant [22] and also the twin image elimination introduced by Koren et al. [26] are frequently suffering from slow convergence and stagnation, they can provide exact phase recovery. That is why we do not want to apply phase retrieval for hologram segmentation, as it was suggested in some earlier approaches [28, but introduce a new, non-iterative hologram segmentation method.

On the other hand, we usually record a hologram of several objects, what we call a composite hologram. That is, the recorded hologram can be considered as the sum of the in-line holograms of the component objects. Higher order (multiple) diffractions [16, 18, 27, 28], as they are very small, can be usually neglected.

Unfortunately, the conventional twin image elimination algorithms generally can not be applied in the case of composite holograms. The spatial (non-negativity, finite support) constraint based phase estimation cannot couple solely to the amplitude of the hologram of a given object, but only to the amplitude of the overall hologram. Therefore, the algorithm generally fails to converge to the correct complex wave field of the object (see Section 4).

Notwithstanding, there were attempts to eliminate the twin image noise in composite holograms by iterative estimation of the objects [23]. However, this algorithm appears to work only for small, distant objects that have mainly amplitude modulation (e.g. particle imaging). It is relatively easy to determine the exact support for such an object and only a small fraction of the energy of the virtual object (twin image) diffracts within this support. In this case, the elimination of the twin image is straightforward and certainly does not require the application of an iterative algorithm $[22,29]$. The phase retrieval algorithms usually converge much slower if the twin image are significant within the support. This is the case of holograms constituted of extended, not sufficiently distant objects that frequently leads 
to inadequate estimation of the supports. Nonetheless, in practical applications, commonly, holograms of just these types of objects are measured [30]. Especially, this is the case when high resolution is aimed.

Even if the own twin image noise of the object does not significantly bias the reconstruction, which is the situation for small, distant objects, the diffraction of the other objects and

their corresponding twin images can still contaminate it considerably. Apart from the twin image noises, this is true even in the case of off-axis holograms.

To avoid this deficiency of the composite hologram reconstruction, the segmentation of the original hologram to the in-line sub-holograms of the constituent objects seems indispensable [31]. The reconstructions based on the segmented holograms will not be biased by the diffractions of the other component objects anymore. However, as we do not know the exact wave field of the objects, this segmentation appears to be hard.

In this paper we introduce a simple, non-iterative algorithm that can segment an in-line hologram to the sum of same sized sub-holograms of the constituent objects, without resolution loss. In contrast to the phase retrieval or twin image elimination algorithms we do not aim or require to reconstruct the complex wave field of all the objects, which would be a much harder task, but only provide a good estimate about the contribution of the particular objects to the original hologram. Even if this algorithm produces practically correct segmentation, it still has some small residual systematic error. In the majority of the possible applications this error can be regarded negligible. Furthermore, by the appropriately amended application of the introduced algorithm the systematic part of the error can be completely eliminated.

In Section 2, we introduce the proposed algorithm. In Section 3, we demonstrate the performance of the algorithm on simulated and measured holograms. We examine the achievable accuracy of the algorithm depending on the segmentation parameters and on their inaccuracies (Section 3.A. In Section 4, we show what kind of technique can be used to eliminate the residual systematic error of the algorithm. It seems essential, when this small systematic error can make the aimed further processing hard (e.g. phase retrieval algorithms). Furthermore, we show that the introduced technique sometimes considerably improves the quality of reconstruction.

\section{In-line hologram segmentation}

The segmentation of the hologram is done object-wise, that is, in every step an in-line approximate hologram of the selected object is determined in addition to the residual hologram. This way, step by step, the in-line hologram of all the objects will be revealed. We segment the in-line hologram into two sub-holograms of the same sizes. One of them is that of the constituent object defined by the support and the reconstruction distance, while the other 
one is the remaining hologram. This way, all the segmented holograms provide reconstructions at the same resolution as the original one. The effective numeric aperture of the object is decreased only by the usually small supports of the other segmented objects (see Section 3).

To find the sub-holograms corresponding to the constituent objects we have to know at least where these objects are within the recorded volume, and what is their approximate extent 23, 26, 29]. We apply a holographic object detection algorithm to find these parameters, namely the proper reconstruction distance and support of the component object. The property, that the objects usually have distinct reconstruction distances, makes the segmentation easier. These depth keys can be exploited to find and separate the objects efficiently. As details of the object detection method are out of scope of this paper, we only provide a short outline of its operation. Its comprehensive analysis will be presented in a forthcoming article.

To define the support and reconstruction distance of the object we reconstruct the hologram at different distances digitally by simulating the propagation of the wave field of the hologram. We use some local image quality measure (focus measure; e.g. Tenengrad's or energy of the gradient image methods [32]) to detect if the object is in focus. Several alternative focus measures have been analysed and compared earlier 32 for this purpose in the case of off-axis holograms. However, twin image noise can deteriorate the success of the earlier proposed autofocusing algorithms, as these diffractions can produce false extremes of the focus measure. We can avoid this problem by the proper application of the in-line hologram segmentation method introduced here.

For digital emulation of wave field propagation we used the angular spectrum method 33]. This method provides the exact solution of the Helmholtz equation describing the free-space propagation of light and it has a straightforward, simple and efficient digital implementation. In contrast to the frequently used Fresnel transform, it does not apply any approximations regarding the reconstruction distance. This way it provides correct wave field evaluation for relatively short propagation distances as well, which is essential in tasks, when high resolution reconstructions are aimed (when the objects should have large effective numeric apertures at the hologram). By its proper application, the numeric errors of the large distance propagations can be controlled 34 . To simulate a single propagation step, the angular spectrum method uses a Fourier transformation, a point-wise complex array multiplication and an inverse Fourier transformation step (Eq. 1). This way it can be implemented on computers efficiently. Its digital implementation can be considerably accelerated by using GPUs [35, 36.

Here, in the in-line hologram segmentation algorithm, we apply only the outputs of the object detection: the estimations of the reconstruction distance and the support of objects. Let us assume that we have these data for all the objects. In Section 3.A we will show that 
even a coarse approximation of these parameters is sufficient for the correct operation of the algorithm. In the case of composite holograms, the supported part of a particular object reconstruction encloses all the information about this object. Obviously it is contaminated by the noise of the twin image and also by the diffractions of the other objects. Out of the support, just these diffractions dominate the reconstructed field and therefore, it can not be used to estimate the object in any way. The essence of the proposed hologram segmentation is to find an in-line hologram, which has the same reconstructed complex amplitude distribution as the original hologram has within the support. Obviously, we have to ensure the consistency of this in-line hologram that is, the supported and unsupported parts have to satisfy the inherent constraints of the in-line hologram structure. For a first approximation, we cannot provide any better estimation of the in-line hologram of the corresponding object than the reconstruction based one. The presence of the diffractions of the other objects within the supported part of the reconstructed wave field will bias the proposed segmentation. Assuming that the segmentations of high contrast objects precede those of the others, their diffractions will not deteriorate the consecutive object segmentations. The refinement of the algorithm will be discussed in Section 4 .

If we illuminate an object of opacity $o(x, y)$ by a monochromatic plane wave of unit amplitude, the scattered field at distance $d$ can be expressed as $h_{d}(1-o)$.

The opacity function corresponds to the amplitude and phase modulation caused by the object, according to its complex refractive index distribution [23]. Here $h_{d}($.$) denotes the d$ distance wave field propagation operation that can be calculated using the angular spectrum method 33]:

$$
h_{d}(E(x, y))=\mathcal{F}^{-1}\left\{\mathcal{F}\{E(x, y)\} e^{i k_{z}(u, v) d}\right\},
$$

where $\mathcal{F}$ and $\mathcal{F}^{-1}$ denote the direct and inverse Fourier transform operations, while

$$
k_{z}(u, v)= \begin{cases}\frac{2 \pi}{\lambda} \sqrt{1-(\lambda u)^{2}-(\lambda v)^{2}}, & \text { if } 1-(\lambda u)^{2}-(\lambda v)^{2}>0 \\ 0, & \text { otherwise. }\end{cases}
$$

The $u$ and $v$ symbols denote the Fourier frequencies in the $x$ and $y$ directions respectively (the illuminating wavelength is $\lambda$ ). This way the scattered field can be expressed as:

$$
h_{d}(1-o)=e^{\frac{i 2 \pi d}{\lambda}}-h_{d}(o)=e^{\frac{i 2 \pi d}{\lambda}}\left(1-h_{d}(O)\right),
$$

where the introduced $O=e^{\frac{-i 2 \pi d}{\lambda}} O$ is still clearly determined by the opacity of the object. The intensity of the wave field at the detector plane (the hologram) is

$$
H=\left|1-h_{d}(o)\right|^{2}=1-h_{d}(O)-\left(h_{d}(O)\right)^{*}+\left|h_{d}(O)\right|^{2} \approx 1-2 \Re\left(h_{d}(O)\right),
$$

where we neglected the second order term, as in the case of Gabor holograms $|o| \ll 1$ is assumed. 
Here we consider composite holograms:

$$
H=\left|1-\sum_{j=1}^{n} h_{d_{j}}\left(o_{j}\right)\right|^{2}
$$

where $o_{j}$ and $d_{j}$ denote the component objects and their distance from the hologram plane, respectively. As multiple diffraction and higher order terms can be neglected, we get

$$
H \approx 1-\sum_{j=1}^{n} h_{d_{j}}\left(O_{j}\right)-\sum_{j=1}^{n}\left(h_{d_{j}}\left(O_{j}\right)\right)^{*}=1-2 \sum_{j=1}^{n} \Re\left(h_{d_{j}}\left(O_{j}\right)\right) .
$$

Let us segment the sub-hologram of the first object, knowing its support $(S)$ and reconstruction distance $\left(d_{1}\right)$ !

$$
H \approx 1-2 \Re\left(h_{d_{1}}\left(O_{1}\right)\right)+H_{r e m},
$$

where $H_{\text {rem }}=-2 \sum_{j=2}^{n} \Re\left(h_{d_{j}}\left(O_{j}\right)\right)$ denotes the contribution of the other objects to the hologram. If we propagate the wave field of the hologram to $\left(-d_{1}\right)$ distance, the result is

$$
I=h_{-d_{1}}(H)=e^{-\frac{i 2 \pi d}{\lambda}}-O_{1}-h_{-2 d_{1}}\left(O_{1}^{*}\right)+h_{-d_{1}}\left(H_{r e m}\right) .
$$

We sucessfully reconstructed the object $\left(O_{1}\right)$ beside the twin image $\left(h_{-2 d}\left(O_{1}^{*}\right)\right)$ and the diffractions caused by the other objects $\left(h_{-d_{1}}\left(H_{r e m}\right)\right)$. Here we used the identity $\left(h_{d}(O)\right)^{*}=$ $h_{-d}\left(O^{*}\right)$. As the last term is significant only outside the support of the first object in most cases, we can simply remove its contribution by filling the unsupported part of the reconstructed hologram by the estimated background (the reference). We get

$$
I^{\prime}=e^{-\frac{i 2 \pi d}{\lambda}}-O_{1}-\left[h_{-2 d_{1}}\left(O_{1}^{*}\right)\right]_{S},
$$

where

$$
[E(x, y)]_{S}= \begin{cases}E(x, y), & \text { if }(x, y) \in S \\ 0, & \text { otherwise }\end{cases}
$$

$O_{1}=\left[O_{1}\right]_{S}$ by definition, and this way $I^{\prime}$ provides a correct estimation of the first object, but it is still biased by the supported part of the twin image $\left(T_{S}=\left[h_{-2 d_{1}}\left(O_{1}^{*}\right)\right]_{S}\right)$. We can use this estimation to determine the contribution of the first object to the hologram. If propagate $I^{\prime}$ to the hologram plane, we get:

$$
h_{d_{1}}\left(I^{\prime}\right)=1-h_{d_{1}}\left(O_{1}\right)-h_{d_{1}}\left(T_{S}\right) .
$$

Considering Eq. 6, we subtract $2\left(\Re\left(h_{d_{1}}\left(I^{\prime}\right)\right)-1\right)$ from the original hologram. Although the first object related term has been successfully eliminated, the supported part of the twin image still biases the remaining hologram:

$$
H^{\prime}=H-\left(2 \Re\left(h_{d_{1}}\left(I^{\prime}\right)\right)-2\right)=1+H_{r e m}+2 \Re\left(h_{d_{1}}\left(T_{S}\right)\right) .
$$


We can recognize that this bias term can be regarded as an in-line hologram of $T_{S}$, with the same reconstruction distance $d_{1}$ and support $(S)$ as $O_{1}$ has. Using the similar method as described above: propagating the estimated remaining hologram to the object plane $\left(d_{1}\right)$ and filling the unsupported part by the background we get:

$$
I^{\prime \prime}=e^{-\frac{i 2 \pi d}{\lambda}}+T_{S}+\left[h_{-2 d_{1}}\left(T_{S}^{*}\right)\right]_{S},
$$

where we neglect again the diffractions of the other objects within the support. Using the identity $\left[h_{-2 d_{1}}\left(T_{S}^{*}\right)\right]_{S}=h_{-2 d_{1}}\left(T_{S}^{*}\right)-\left[h_{-2 d_{1}}\left(T_{S}^{*}\right)\right]_{\bar{S}}$ (where $[E]_{\bar{S}}$ clears the unsupported part of $E)$ and propagating this modified reconstruction to the hologram plane we get:

$$
h_{d_{1}}\left(I^{\prime \prime}\right)=1+h_{d_{1}}\left(T_{S}\right)+h_{-d_{1}}\left(T_{S}^{*}\right)+\varepsilon=1+2 \Re\left(h_{d_{1}}\left(T_{S}\right)\right)+\varepsilon,
$$

where $\varepsilon=-h_{d_{1}}\left[h_{-2 d_{1}}\left(T_{S}^{*}\right)\right]_{\bar{S}}$. This way we can eliminate the bias of the supported twin image:

$$
H^{\prime \prime}=\Re\left(H^{\prime}-\left(h_{d_{1}}\left(I^{\prime \prime}\right)-1\right)\right)=1+H_{r e m}-\Re(\varepsilon),
$$

while the segmented object contribution to the in-line hologram (considering (Eq. 5).) is defined:

$$
H_{1}=H-H^{\prime \prime}=-2 \Re\left(h_{d_{1}}\left(O_{1}\right)\right)+\Re(\varepsilon) .
$$

The remaining segmentation error is:

$$
-\Re(\varepsilon)=\Re\left(h_{d_{1}}\left[h_{-2 d_{1}}\left(T_{S}^{*}\right)\right]_{\bar{S}}\right)=\Re\left(h_{d_{1}}\left[h_{-2 d_{1}}\left[h_{2 d_{1}}\left(O_{1}\right)\right]_{S}\right]_{\bar{S}}\right) .
$$

That is, we propagate the wave field of $O_{1}$ to $2 d_{1}$ and erase the unsupported part; then propagate back the result by $\left(-2 d_{1}\right)$ and erase the supported part. If we did not erase the unsupported part, the result would be by definition zero. Therefore, it is a reasonable assumption that the remaining error is really small. The size of the remaining error, however depends considerably on the properties of the applied support.

If there is no information about the amplitude of the background illumination, then we can use the reconstructed hologram to estimate it. The phase of the background is defined by the actual reconstruction distance $\left(-2 \pi d_{1} / \lambda\right)$, while its amplitude is estimated - due to the potentially uneven illumination - from the measured local mean amplitude.

To decrease further the diffraction on the support boundaries we use Gaussian smoothing 23] (using standard deviation of 2 pixels). Worth to note, that the remaining support boundary diffractions can considerably limit the achievable accuracy of the proposed algorithm and might lead to some object- and support-related systematic error (Section 3.A.).

On the other hand, this remaining unsupported systematic bias can not be erased by the repetition of the algorithm. There are other sources of biases like the diffraction on the support boundaries, the supported part of the diffractions of the other objects and the 
neglected higher order diffraction terms. As there is no simple way to eliminate these biases, it is not reasonable to try to diminish further the remaining unsupported, small noise term. Notwithstanding, we can consider applying an iterative method which tries to eliminate the amplitude modulation within the supported region not only at the object plane, but at the virtual object plane too. Due to the different types of noises mentioned above, the correct convergence of such an algorithm seems dubious.

\section{Summing up the proposed algorithm:}

- First, reconstruct the object by the simulated propagation of the hologram.

- Fill the unsupported part of the reconstructed object with the background.

- Propagate this wave field to the hologram plane.

- Subtract twice the real part of the result from the original hologram and add two times the absolute value of the background.

- Propagate this estimated remaining hologram wave field to the object plane.

- Fill the unsupported part of the reconstruction with the background.

- Propagate this wave field to the hologram plane, subtract it from the estimated remaining hologram and add the absolute value of the background. The real part of the result is the segmented remaining hologram.

Algorithmic steps and operational principles of the introduced hologram segmentation are explained in Fig. 2 ,

\section{Results and analysis}

The introduced hologram segmentation method, contrary to the earlier approaches, works not only for (small, distant) amplitude objects, as it was depicted in the introduction, but also for extended objects with complex opacity functions. Furthermore, the algorithm provides much higher speed as it is constructed to be non-iterative. To demonstrate the segmentation algorithm performance we show its effects on a measured hologram. In the next image (Fig. 3) it can be seen that using the proposed algorithm the original hologram is segmented to an in-line sub-hologram and to the residual hologram. There is no perceptible, apparent error of this segmentation. The diffraction patterns of both the segmented object and the objects of the remaining hologram are neatly preserved. In the next image (Fig. 4) we demonstrate the algorithm function in a real world task, segmenting a measured composite hologram, where the sample objects were freely floating algae within a large volume of water. In the attached 
short video (Media 1) we can see how the algorithm defines and eliminates the contribution of the different objects to the original hologram in succession. This way, the diffractions of the segmented objects are removed from the hologram and do not disturb the reconstruction of the last object (Asterionella alga) considerably. Worth to note, that although a small part of the recorded hologram was displayed, we used the original, high resolution holograms in the segmentation and reconstruction tasks, therefore the segmentation does not result is considerable resolution loss.

As the introduced hologram segmentation removes all the modulations within the support at a given object reconstruction distance, it would remove the diffractions of the other objects here, as well. These diffractions would be missing from the holograms of the actual objects and this way their reconstruction is biased. That is, the segmentation of the objects decrease the apertures of the not yet segmented ones, and slightly decrease their achievable resolution. These missing terms cause perceivable reconstruction bias only in the case of nearby, severely overlapping objects. However, this error can be regarded negligible, if we consider sparse and small sample objects as it is usually assumed in Gabor holograms.

\section{A. Analysis}

To analyze the in-line hologram segmentation algorithm performance we have to estimate its remaining error considering different parameters and their biases. We used a simulated hologram of a single object because in this case the expected result of the segmentation is exactly known. The error is estimated by the relative energy of the residual modulation of the hologram comparing to that of the original hologram one according to Eq. (16).

$$
E=\frac{\sum_{(x, y)}\left(H^{\prime \prime}(x, y)-<H^{\prime \prime}(x, y)>\right)^{2}}{\sum_{(x, y)}(H(x, y)-<H(x, y)>)^{2}}
$$

In the next figure (Fig. 5(a)) the remaining error can be seen as a function of the reconstruction distance. It can be recognized that at large distances the error decreases with the distance as the supported part of the object reconstruction approximates the original object better (the supported part of the twin image decreases). For small distances as more and more energy of the object and the twin image falls within the support, the relative residual error of the algorithm decreases (Fig. 5(a)), because the algorithm extincts the modulation just here.

The algorithm appears to be robust against the poor approximation of the reconstruction distance parameter (Fig. 5(b)). For relatively large biases of the reconstruction distance the algorithm can still produce fairly correct segmentation.

The residual error decreases with the increasing support size, as it is expected (Fig. 5(c)). 
However, as the applied support decreases the aperture of the other objects some way, we cannot increase its size without penalty. Furthermore, the other objects confine the achievable size of the support, because the supports cannot overlap. If the applied support is too large, it can envelop more of the diffractions of the other objects and support boundaries can cause larger diffractions.

We tested the effects of different support shapes on the residual error of the segmentation (Fig. 6). It can be recognized that the algorithm is really sensitive to the applied support (Fig. 6(a) and (b)). This result indicates that the diffractions on the support boundaries cause the majority of the residual error. We tested it using several different supports and averaged the obtained segmented holograms (as in Fig. 6(c)). These results show that the residual error of the single support segmentation ( 0.0026) noticeably decreased (0.0008). Although, this method does not provide an efficient way to erase the residual error, as it requires numerous executions of the algorithm applying different supports, it explains its origin. This error is small close to the applied reconstruction distance and probably does

not bias the reconstruction of the nearby objects. Worth to note, that the diffraction on the support boundaries appears also in the phase retrieval algorithms, and only the iteratively removed twin image can erase it.

To be able to estimate the size of this residual error we computed the otherwise neglected second order term of the object (the fourth term in Eq. (3)). In the case of a relatively small reconstruction distance, it appears to be comparable to the segmentation error (e.g. if the reconstruction distance is $5 \mathrm{~mm}$ then the measured segmentation noise is 0.00459 , while the second order term noise is 0.00553$)$.

\section{Application of the segmentation results}

The question is whether the small systematic (mainly support-dependent) error of the segmentation can disallow the efficient application of this method. Here we consider how to apply this method in phase retrieval and object reconstruction algorithms.

Phase retrieval is required if we intend to reconstruct the exact phase distribution of the object field. It is necessary when approximated shape or thickness information is to be obtained 11, 12. The problem is that the segmented hologram has some support related small remaining error that can inhibit the proper application of the phase retrieval algorithms. We can avoid this by the special, amended application of the segmentation results. We can get a correct approximation of the hologram of a given object, if we subtract the segmented holograms of all the other objects from the measured original hologram. It will not be biased by any systematic error as the supported and unsupported object related terms remain consistent. As we use all the segmentation results, it will accumulate the errors of the hologram segmentations of the particular objects. These errors, however, can be regarded as a small, 
relatively distant (out of support) noise. That is, they do not cause special inconsistencies of the supported and unsupported parts of the corresponding hologram of a given object. This way the phase retrieval algorithms become applicable in the case of composite holograms.

To demonstrate the applicability of the introduced hologram segmentation method for phase retrieval we compared the Gerchberg-Saxton algorithm performance on a synthetic composite hologram. We constructed a synthetic, composite in-line hologram of two simple sample objects that have small tilted phase modulation and different 3D positions. As the applied test object was complex, its opacity function has some clearly visible amplitude modulation (especially, where it is higher than background amplitudes).

In Fig. 7we can see that the reconstruction of the first object from the composite hologram is severely contaminated by the diffractions of the other objects. These diffractions can be eliminated by the application of the corrected hologram segmentation. It can be seen that the Gerchberg-Saxton algorithm does not converge in the case of a composite hologram, while it works appropriately for the correctly segmented hologram. Worth to note, that the apparently slow or missing convergence of the phase retrieval algorithms make it uncertain if they can be applied efficiently in general for twin image elimination.

The applied phase retrieval method was able to reconstruct the complex opacity function of the test object (Fig. 7). Actually, the precision (and speed) of phase retrieval depends considerably on the tightness of the applied support. As it was deliberately choosen to be loose, the exact phase retrieval had not been achieved.

If the retrieval of the exact phase information is not critical, even a simple object reconstruction can be acceptable. However, as we discussed in the Section 1, the diffraction of some other objects can deteriorate the reconstruction of the segmented hologram of a given object. This frequently occurs, but usually the distortion is small or negligible. However, in special (3D) positions of the objects this diffraction caused noise can be considerable. Applying the above mentioned corrected hologram segmentation method that is subtracting all but one segmented hologram from the measured one, all the diffractions of the other objects will be minimized within the reconstructed object support. In the next figure (Fig. 8), we can compare the reconstruction quality of a scenedesmus alga based on the combined, the segmented and the correctly segmented holograms. (The measured, large, high resolution hologram made it possible to achieve reconstructions of $\lesssim 1 \mu \mathrm{m}$ resolution.)

\section{Conclusion}

We introduced a non-iterative in-line composite hologram segmentation algorithm. It can provide a high-quality estimation of the hologram of a particular object in the original hologram. It requires only four simple propagation steps that can be implemented efficiently on a computer, using the angular spectrum method. As our algorithm is non-iterative, it 
is hard to compare to those ones, that provide exact phase retrieval of all the objects. They require much more computation and can be applied only in the case of distant (pure amplitude) objects, when the achievable resolution of the reconstructions is severely confined. We analyzed the algorithm performance and showed how the remaining error depends on the applied parameters and their accuracies. We confirmed that the vast majority of the

otherwise small residual error is caused by the diffractions on the support boundaries. It is also shown how the segmented holograms can be applied efficiently in phase retrieval or object reconstruction tasks. The proposed algorithm can be used in other hologram processing, preprocessing steps, where twin image noise and diffractions of the other constituent objects do not allow to achieve correct results.

\section{Acknowledgments}

This work was funded by the Hungarian National Office for Research and Technology (NKTH 1981822A) project entitled Water Biology Digital Holographic Microscope (DHM) as an early warning environmental system.

\section{References}

1. D. Gabor, "A new microscopic principle," Nature 161, 777-778 (1948).

2. J. Burns, N. Watson, "Data extraction from underwater holograms of marine organisms," OCEANS 2007 - Europe 1, 1-6 (2007).

3. P. Marquet, B. Rappaz, T. Colomb, F. Charrière, J. Kühn, Y. Emery, E. Cuche, C. Depeursinge, and P. Magistretti, "Digital holographic microscopy, a new optical imaging technique to investigate cellular dynamics," Proc. SPIE 6191 (2006).

4. J. Hahn, S. Lim, K. Choi, R. Horisaki, and D. Brady, "Video-rate compressive holographic microscopic tomography," Optics Express 19, 7289-7298 (2011).

5. P. Langehanenberg, G. von Bally, and B. Kemper, "Autofocusing in digital holographic microscopy," 3D Research 2, 1-11 (2010).

6. M. DaneshPanah and B. Javidi, "Tracking biological microorganisms in sequence of 3d holographic microscopy images," Optics Express 15, 10761-10766 (2007).

7. Z. Göröcs, L. Orzó, M. Kiss, V. Tóth, and S. Tőkés, "In-line color digital holographic microscope for water quality measurements," Proc. SPIE 7376, 737614 (2010).

8. Z. Göröcs, M. Kiss, V. Tóth, L. Orzó, and S. Tőkés, "Multicolor digital holographic microscope (dhm) for biological purposes," Proc. SPIE 7568, 75681P (2010).

9. F. Dubois, C. Yourassowsky, O. Monnom, J. Legros, O. Debeir, P. Van Ham, R. Kiss, and C. Decaestecker, "Digital holographic microscopy for the three-dimensional dynamic analysis of in vitro cancer cell migration," Journal of Biomedical Optics 11, 054032 (2006). 
10. T. Colomb, F. Charrière, J. Kühn, P. Marquet, and C. Depeursinge, "Advantages of digital holographic microscopy for real-time full field absolute phase imaging," Proc. SPIE 6861, 1-10 (2008).

11. J. Kühn, F. Charrière, T. Colomb, E. Cuche, F. Montfort, Y. Emery, P. Marquet, and C. Depeursinge, "Axial sub-nanometer accuracy in digital holographic microscopy," Measurement Science and Technology 19, 074007 (2008).

12. P. Marquet, B. Rappaz, P. Magistretti, E. Cuche, Y. Emery, T. Colomb, and C. Depeursinge, "Digital holographic microscopy: a noninvasive contrast imaging technique allowing quantitative visualization of living cells with subwavelength axial accuracy," Optics letters 30, 468-470 (2005).

13. A. Stern and B. Javidi, "Theoretical analysis of three-dimensional imaging and recognition of micro-organisms with a single-exposure on-line holographic microscope," JOSA A 24, 163-168 (2007).

14. O. Matoba, T. Naughton, Y. Frauel, N. Bertaux, and B. Javidi, "Real-time threedimensional object reconstruction by use of a phase-encoded digital hologram," Applied optics 41, 6187-6192 (2002).

15. N. Shaked, Y. Zhu, M. Rinehart, and A. Wax, "Two-step-only phase-shifting interferometry with optimized detector bandwidth for microscopy of live cells," Optics Express 17, 15585-15591 (2009).

16. J. Garcia-Sucerquia, W. Xu, S. Jericho, P. Klages, M. Jericho, and H. Kreuzer, "Digital in-line holographic microscopy," Applied optics 45, 836-850 (2006).

17. J. Sheng, E. Malkiel, and J. Katz, "Digital holographic microscope for measuring threedimensional particle distributions and motions," Applied optics 45, 3893-3901 (2006).

18. F. Pellistri, C. Pontiggia, L. Repetto, and E. Piano, "Gabor's hologram in a modern perspective," American journal of physics pp. 964-967 (2004).

19. S. Jericho, J. Garcia-Sucerquia, W. Xu, M. Jericho, and H. Kreuzer, "Submersible digital in-line holographic microscope," Review of scientific instruments 77, 043706 (2006).

20. C. Oh, S. Isikman, B. Khademhosseinieh, and A. Ozcan, "On-chip differential interference contrast microscopy using lensless digital holography," Opt. Express 18, 4717-4726 (2010).

21. C. P. McElhinney, B. M. Hennelly, and T. J. Naughton, "Twin-image reduction in inline digital holography using an object segmentation heuristic," Journal of Physics: Conference Series 139, 012014 (2008).

22. B. Hennelly, D. Kelly, N. Pandey, and D. Monaghan, "Review of twin reduction and twin removal techniques in holography," (2009).

23. L. Denis, C. Fournier, T. Fournel, and C. Ducottet, "Numerical suppression of the twin image in in-line holography of a volume of micro-objects," Measurement Science and 
Technology 19, 074004 (10pp) (2008).

24. A. Coskun, I. Sencan, T. Su, and A. Ozcan, "Lensless wide-field fluorescent imaging on a chip using compressive decoding of sparse objects," Optics express 18, 10510 (2010).

25. J. Fienup, "Phase retrieval algorithms: a comparison," Applied Optics 21, 2758-2769 (1982).

26. G. Koren, F. Polack, and D. Joyeux, "Iterative algorithms for twin-image elimination in in-line holography using finite-support constraints," Journal of the Optical Society of America A 10, 423-433 (1993).

27. P. Hariharan, Optical Holography: principles, techniques, and applications, 20 (Cambridge Univ Pr, 1996).

28. L. Denis, C. Fournier, T. Fournel, and C. Ducottet, "Twin-image noise reduction by phase retrieval in in-line digital holography," (2005).

29. C. McElhinney, B. Hennelly, L. Ahrenberg, and T. Naughton, "Removing the twin image in digital holography by segmented filtering of in-focus twin image," Proc. of SPIE 7072, 7 (2008).

30. W. Bishara, T. Su, A. Coskun, and A. Ozcan, "Lensfree on-chip microscopy over a wide field-of-view using pixel super-resolution," Optics express 18, 11181-11191 (2010).

31. S. Isikman, W. Bishara, S. Mavandadi, F. Yu, S. Feng, R. Lau, and A. Ozcan, "Lens-free optical tomographic microscope with a large imaging volume on a chip," Proceedings of the National Academy of Sciences 108, 7296 (2011).

32. I. Bergoënd, T. Colomb, N. Pavillon, Y. Emery, and C. Depeursinge, "Depth-of-field extension and 3d reconstruction in digital holographic microscopy," Proc. SPIE 7390, 73901C-1 (2009).

33. J. Goodman, Introduction to Fourier optics (Roberts \& Company Publishers, 2005).

34. K. Matsushima and T. Shimobaba, "Band-limited angular spectrum method for numerical simulation of free-space propagation in far and near fields," Optics Express 17, 19662-19673 (2009).

35. T. Shimobaba, Y. Sato, J. Miura, M. Takenouchi, and T. Ito, "Real-time digital holographic microscopy using the graphic processing unit," Optics Express 16, 11776-11781 (2008).

36. L. Orzó, Z. Göröcs, I. Szatmári, and S. Tőkés, "Gpu implementation of volume reconstruction and object detection in digital holographic microscopy," in "Proceedings of IEEE Conference on Cellular Nanoscale Networks and Their Applications (CNNA)," (IEEE, 2010), pp. 1-4. 


\section{List of Figure Captions}

Fig. 1. Optical setup of the in-line DHM (a) makes it possible to measure the hologram of volumetric samples. Although, diffractions of the sample objects frequently overlap in the recorded hologram (b), by the application of proper hologram segmentation we can retrieve the correct images of these objects with their 3D positions.

Fig. 2. Algorithmic steps and operational principles of the introduced hologram segmentation are explained on a synthetic test hologram (a). Supported part (c) of the reconstruction (b) of the composite test hologram defines the first approximation of the segmented and the remaining hologram (d). However, there is a recognizable bias in this approximation, which is caused by the supported part of the twin image. This bias can be retrieved (g) and eliminated from the approximated remaining hologram reconstruction (e), using the property that the reconstruction of the segmented hologram approximation contains effectively no modulation outside the support (f). This way, the original hologram is correctly segmented into a sub-hologram (h) and the remaining hologram (i).

Fig. 3. A measured in-line hologram (a) is segmented to a hologram according to one of the constituent object and to the residual hologram. Although the segmented holograms have the same size and resolution as the original one, to help the observation a zoomed part (b) of the original hologram and the same zoomed parts of the segmented and the remaining holograms are shown $((\mathrm{c})$ and $(\mathrm{d}))$. It can be seen that all the object related diffraction patterns are removed from the remaining hologram, while the interference fringes of the other objects are correctly preserved (scale bars denote $20 \mu \mathrm{m}$ ).

Fig. 4. The segmentation algorithm can eliminate the holographic contribution of the different objects from the measured composite hologram one after the other (Media 1). This way, the diffractions of the segmented objects are removed from the hologram and do not disturb the reconstruction of the last object (Asterionella alga). (a) Measured composite hologram. The sample objects were freely floating algae in a large volume of water, (b) Reconstruction of a given object (a Nitzschia alga) in the approximated reconstruction distance. The crudely estimated support is highlighted, (c) The contribution of this reconstructed object to the original hologram (scale bars denote $20 \mu \mathrm{m}$ ).

Fig. 5. The residual error of the segmentation appears to be small for small and large reconstruction distances, as well (a). The segmentation algorithm is robust against a small bias of the applied reconstruction distance (b), while the residual error decreases with the support size (c) as it is expected. 
Fig. 6. We can recognize that the small residual error of the segmentation algorithm depends on the shape of the support of the object ((a) and (b)). Using the average of several segmented holograms (applying 4 elliptic supports of different orientation) we can reduce this error considerably (c).

Fig. 7. To demonstrate the applicability of the hologram segmentation in phase retrieval tasks we constructed a synthetic composite hologram (a) from two amplitude and phase modulated test objects. The reconstruction of one of the objects is considerably biased by the diffraction of the other object (b). Using the corrected hologram segmentation results we can eliminate the contribution of the second object from the hologram (c) and also from the reconstruction (d). Phase retrieval can not be applied directly in the case of combined in-line holograms. It can be seen that the modified Gerchberg-Saxton algorithm does not converge for the composite hologram. We can recognize even some worsening of the retrieved image quality (see insets). Conversely, the modified Gerchberg-Saxton algorithm (slowly) converges if we apply it on the segmented hologram.

Fig. 8. To find the correct hologram of a particular object (scenedesmus alga) without any systematic error (c) we can subtract all the segmented sub-holograms of the nearby objects from the original measured hologram (a). Diffractions of other objects can bias the reconstruction of the actual object ((b) and (d)), if we use the original (a) or the segmented hologram (c), respectively. Conversely, if we apply the corrected hologram segmentation results (e), these biases are also eliminated (f). Here only zoomed part of the holograms and reconstructions are shown, but the algorithm uses the original, high resolution holograms (scale bars denote $20 \mu \mathrm{m}$ ). 


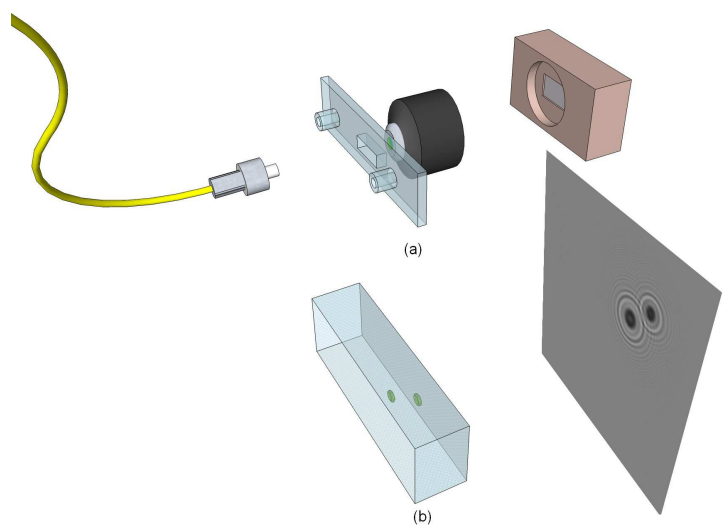

Fig. 1. Optical setup of the in-line DHM (a) makes it possible to measure the hologram of volumetric samples. Although, diffractions of the sample objects frequently overlap in the recorded hologram (b), by the application of proper hologram segmentation we can retrieve the correct images of these objects with their 3D positions. HS10000F1.eps. 


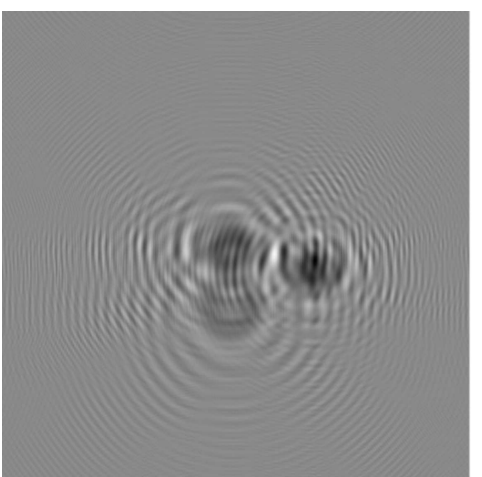

(a)

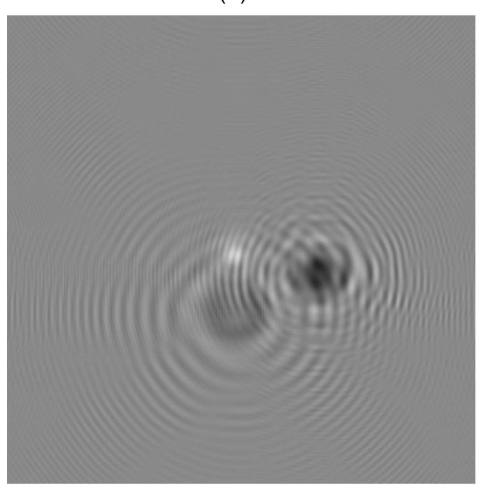

(d)

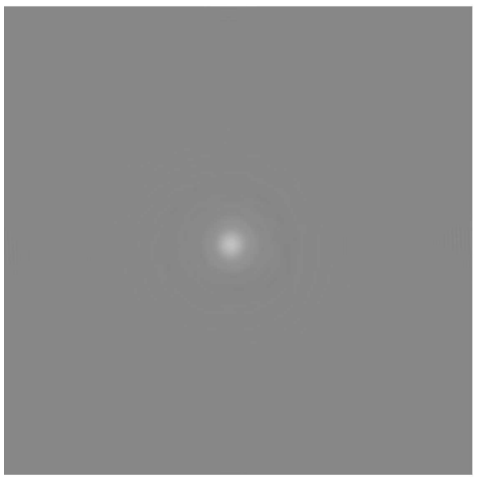

(g)

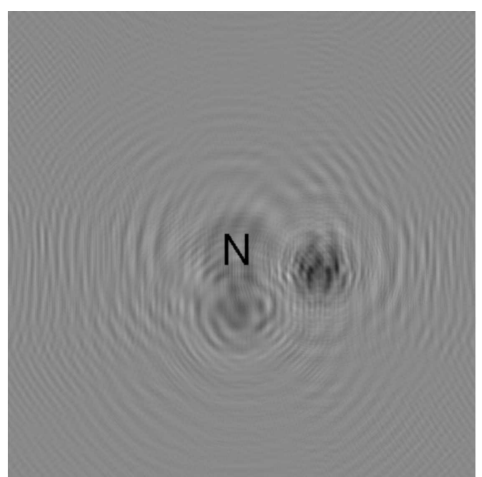

(b)

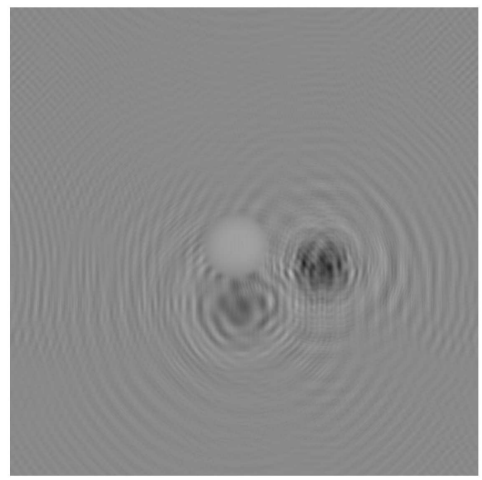

(e)

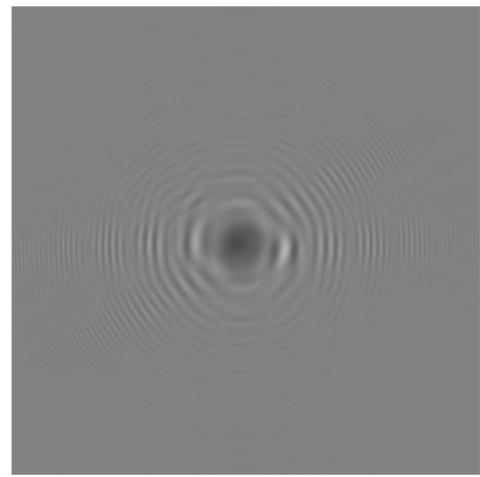

(h)

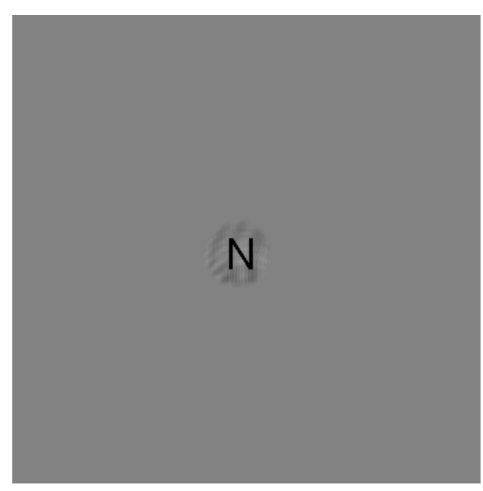

(c)

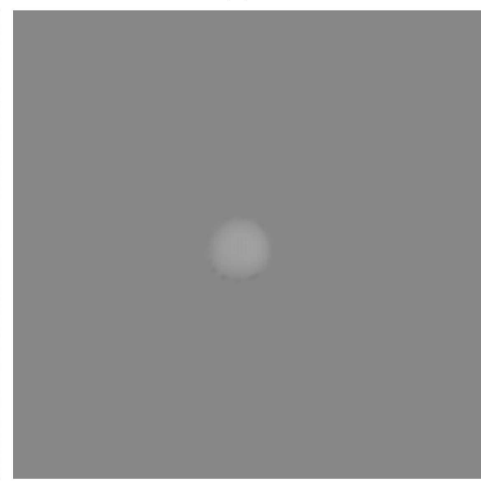

(f)

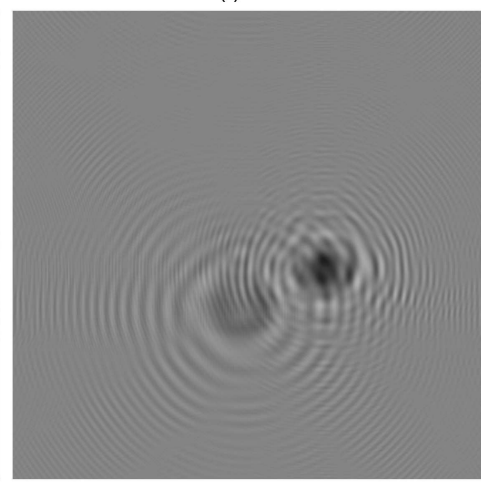

(i)

Fig. 2. Algorithmic steps and operational principles of the introduced hologram segmentation are explained on a synthetic test hologram (a). Supported part (c) of the reconstruction (b) of the composite test hologram defines the first approximation of the segmented and the remaining hologram (d). However, there is a recognizable bias in this approximation, which is caused by the supported part of the twin image. This bias can be retrieved $(\mathrm{g})$ and eliminated from the approximated remaining hologram reconstruction (e), using the property that the reconstruction of the segmented hologram approximation contains effectively no modulation outside the support (f). This way, the original hologram is correctly segmented into a sub-hologram (h) and the remaining hologram (i). HS10000F2.eps. 


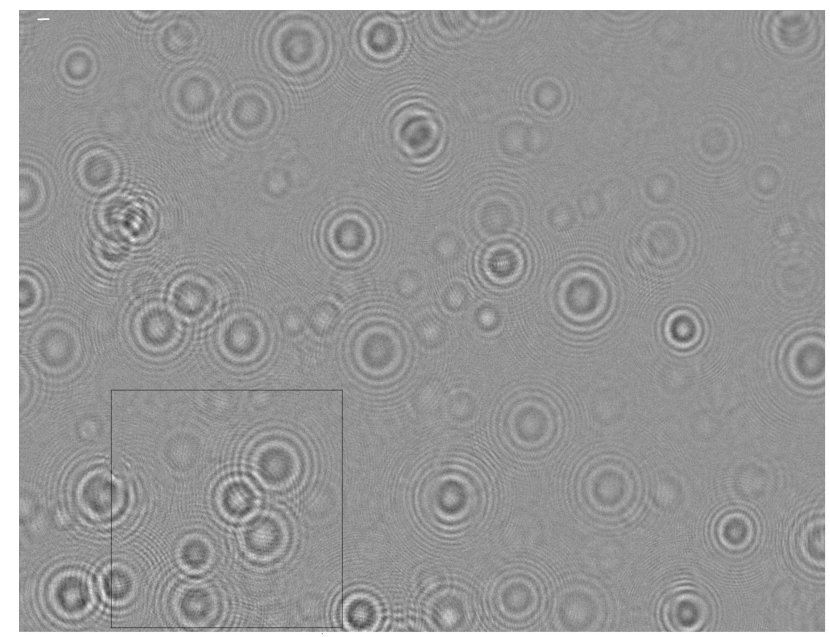

(a)

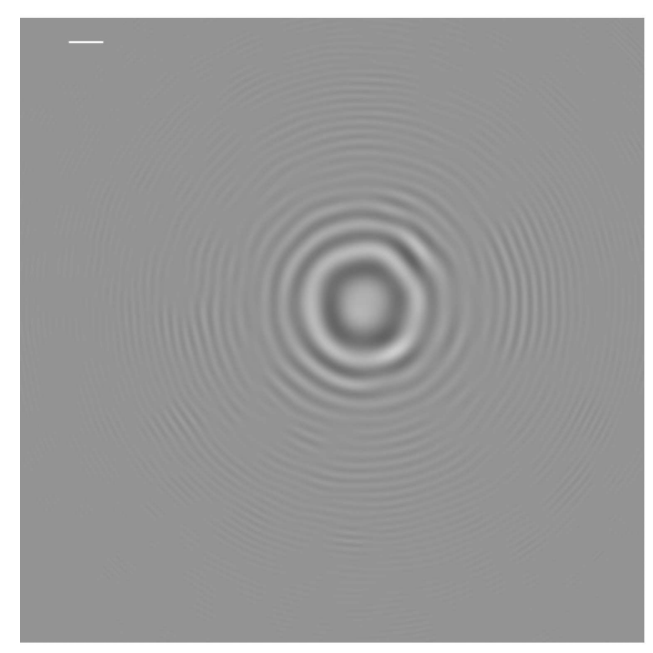

(c)

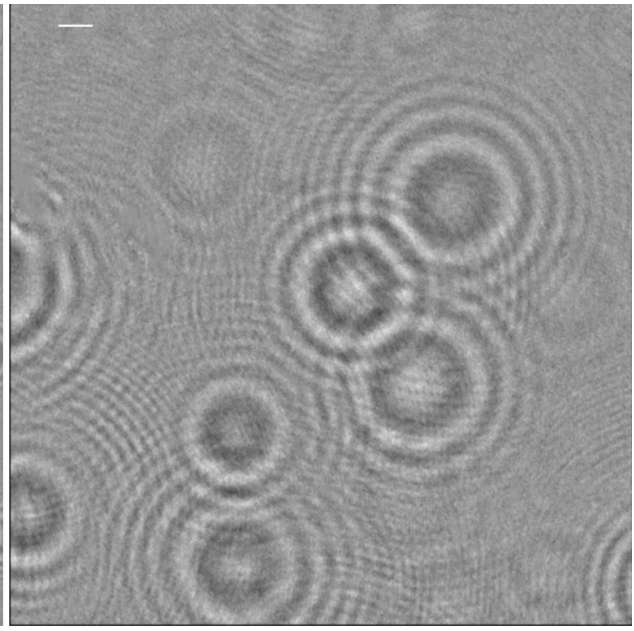

(b)

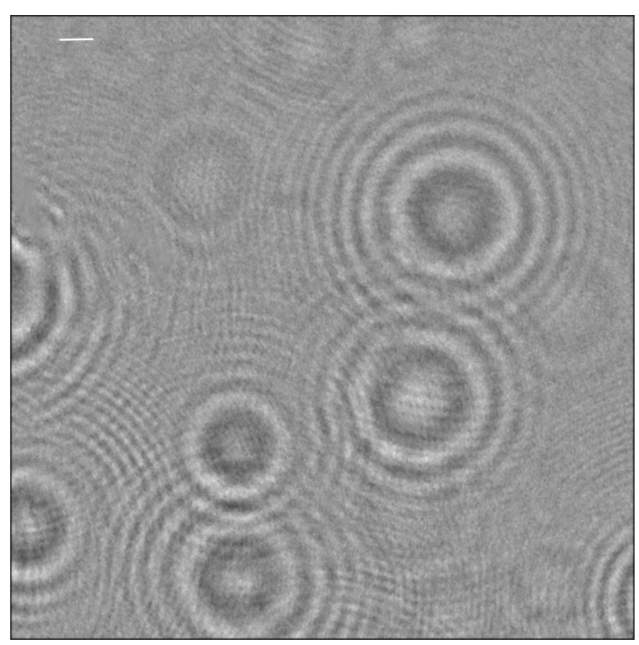

(d)

Fig. 3. A measured in-line hologram (a) is segmented to a hologram according to one of the constituent object and to the residual hologram. Although the segmented holograms have the same size and resolution as the original one, to help the observation a zoomed part (b) of the original hologram and the same zoomed parts of the segmented and the remaining holograms are shown ((c) and $(d))$. It can be seen that all the object related diffraction patterns are removed from the remaining hologram, while the interference fringes of the other

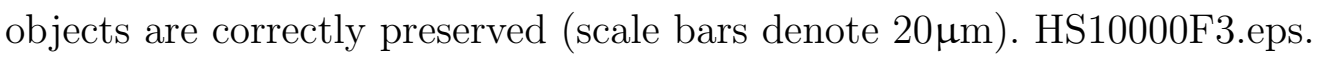



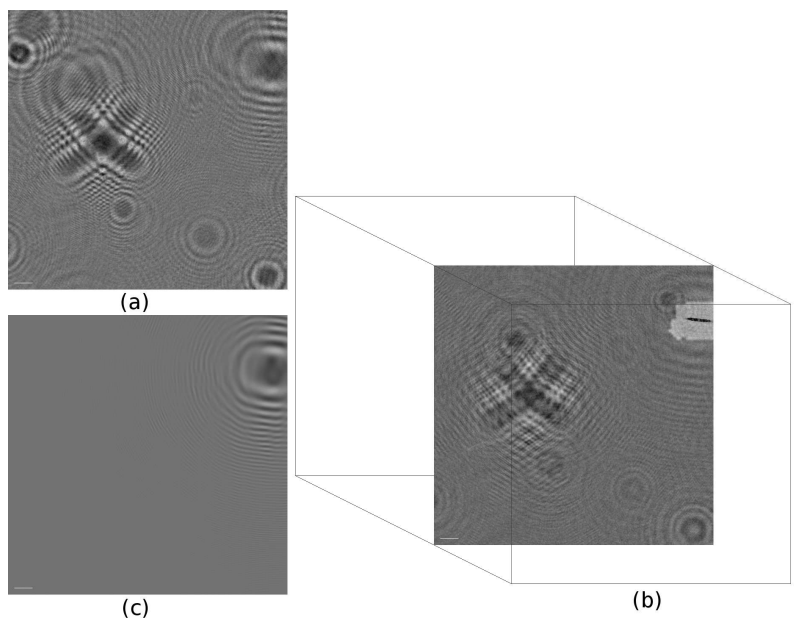

(b)

Fig. 4. The segmentation algorithm can eliminate the holographic contribution of the different objects from the measured composite hologram one after the other (Media 1). This way, the diffractions of the segmented objects are removed from the hologram and do not disturb the reconstruction of the last object (Asterionella alga). (a) Measured composite hologram. The sample objects were freely floating algae in a large volume of water, (b) Reconstruction of a given object (a Nitzschia alga) in the approximated reconstruction distance. The crudely estimated support is highlighted, (c) The contribution of this reconstructed object to the original hologram (scale bars denote $20 \mu \mathrm{m}$ ). HS10000F4.eps. 


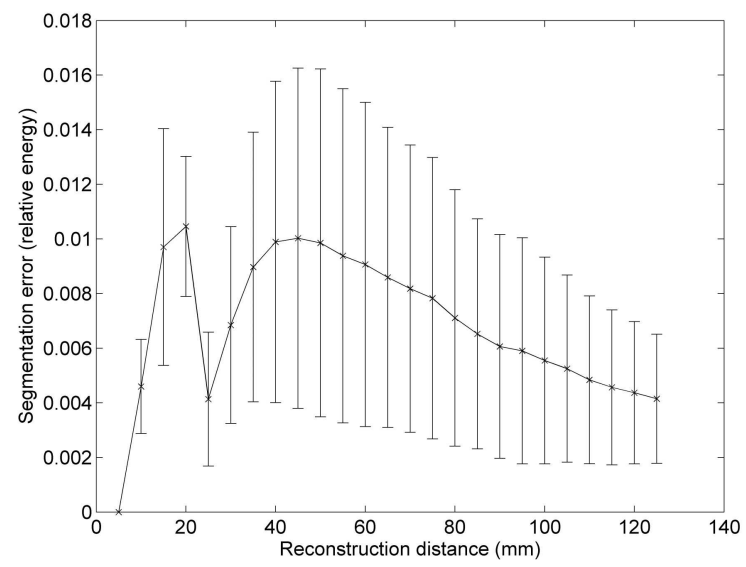

(a)

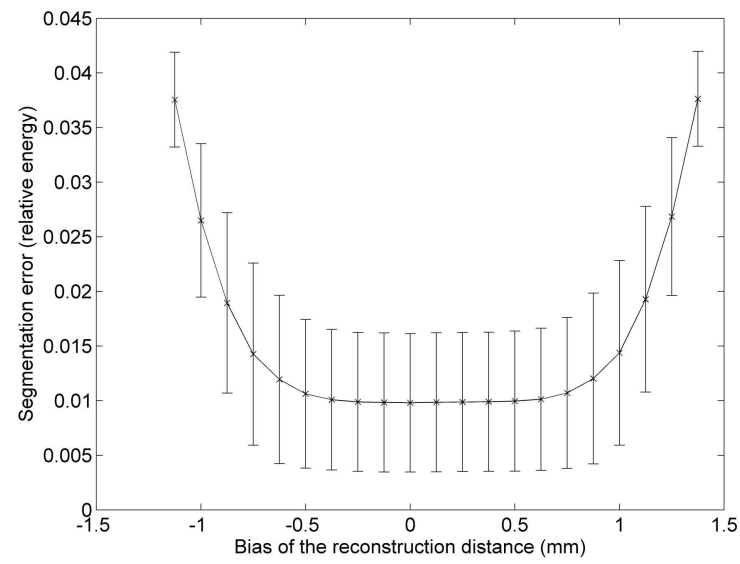

(b)

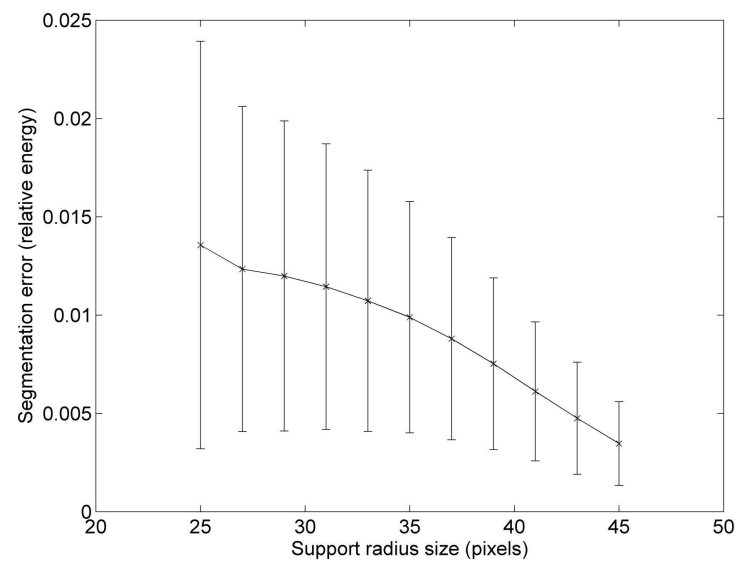

(c)

Fig. 5. The residual error of the segmentation appears to be small for small and large reconstruction distances, as well (a). The segmentation algorithm is robust against a small bias of the applied reconstruction distance (b), while the residual error decreases with the support size (c) as it is expected. HS10000F5.eps. 


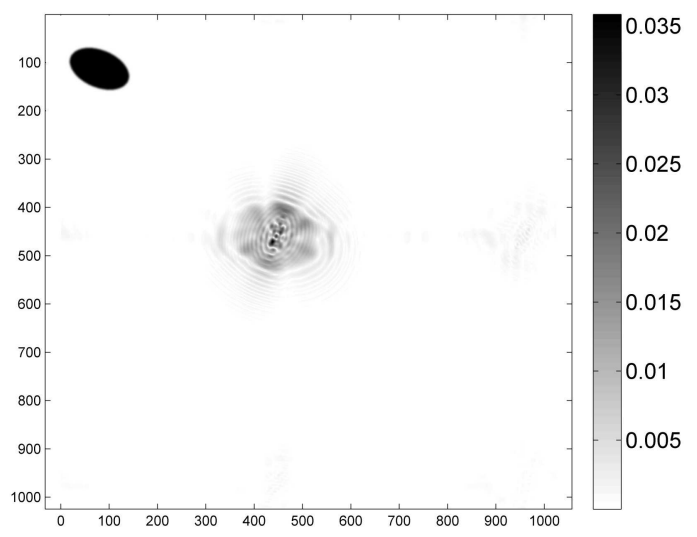

(a)

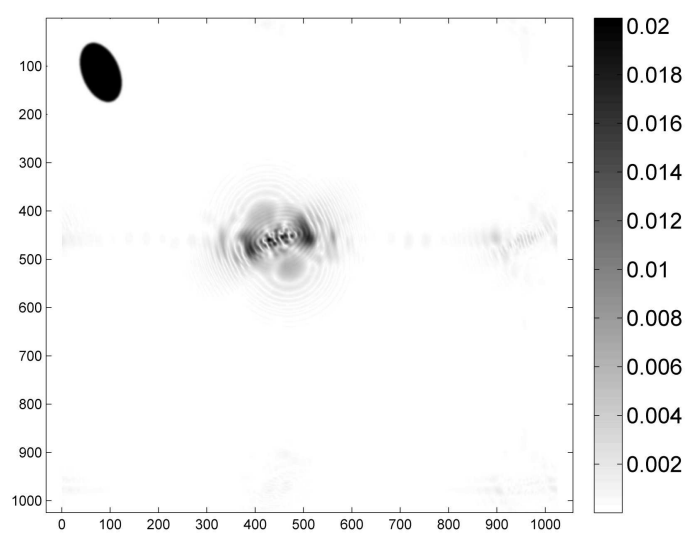

(b)

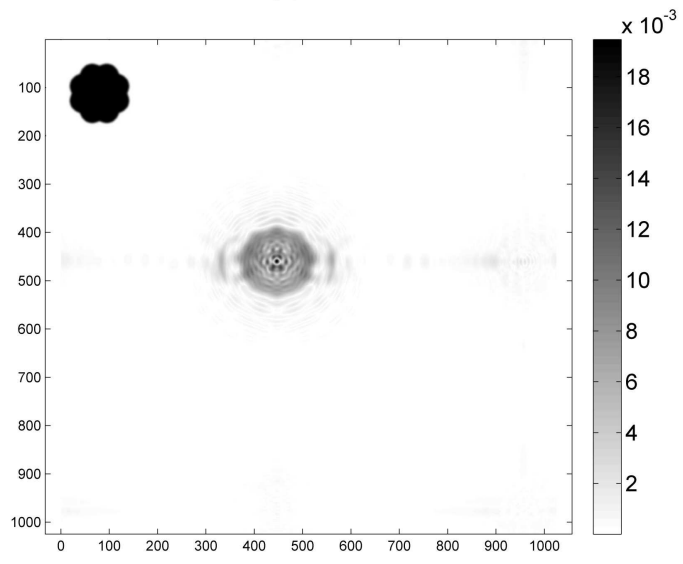

(c)

Fig. 6. We can recognize that the small residual error of the segmentation algorithm depends on the shape of the support of the object ((a) and (b)). Using the average of several segmented holograms (applying 4 elliptic supports of different orientation) we can reduce this error considerably (c). HS10000F6.eps. 


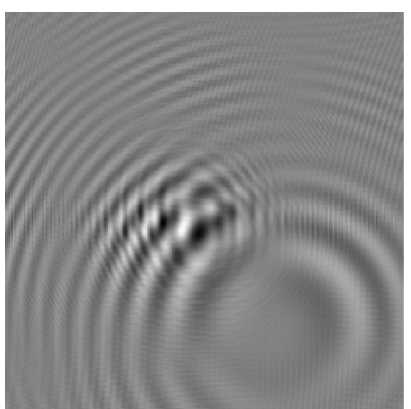

(a)

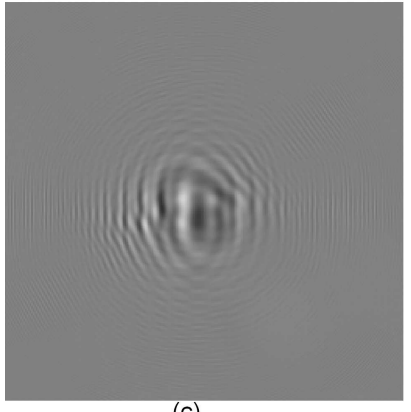

(c)

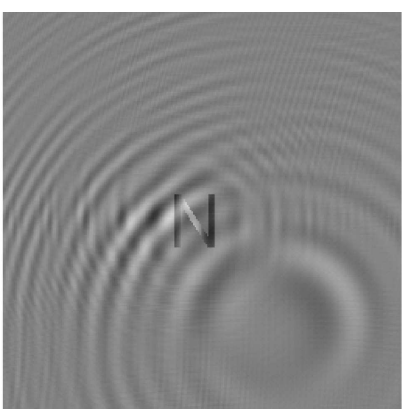

(b)

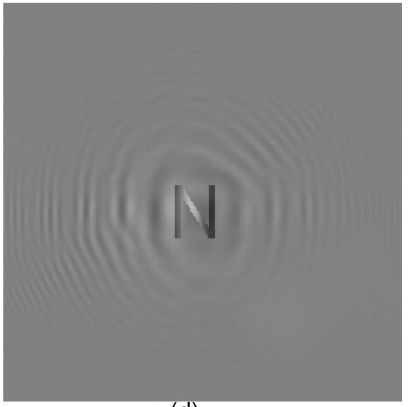

(d)

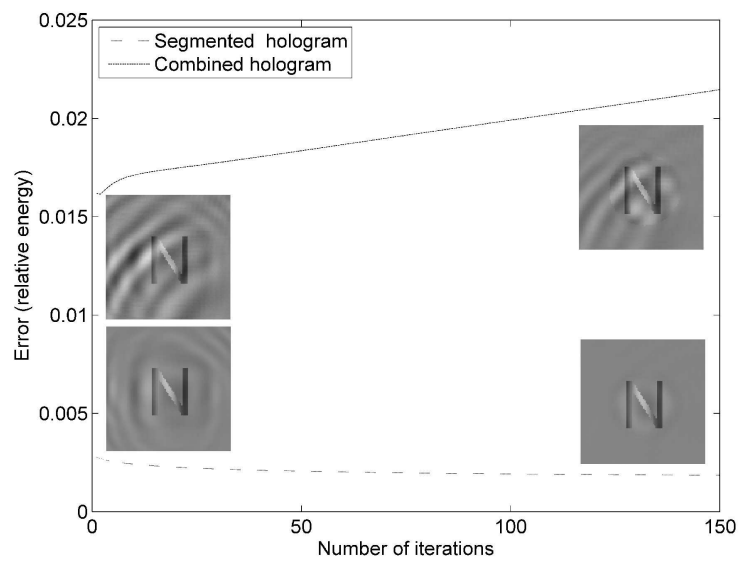

(e)

Fig. 7. To demonstrate the applicability of the hologram segmentation in phase retrieval tasks we constructed a synthetic composite hologram (a) from two amplitude and phase modulated test objects. The reconstruction of one of the objects is considerably biased by the diffraction of the other object (b). Using the corrected hologram segmentation results we can eliminate the contribution of the second object from the hologram (c) and also from the reconstruction (d). Phase retrieval can not be applied directly in the case of combined inline holograms. It can be seen that the modified Gerchberg-Saxton algorithm does not converge for the composite hologram. We can recognize even some worsening of the retrieved image quality (see insets). Conversely, the modified Gerchberg-Saxton algorithm (slowly) converges if we apply it on the segmented hologram. HS10000F7.eps. 


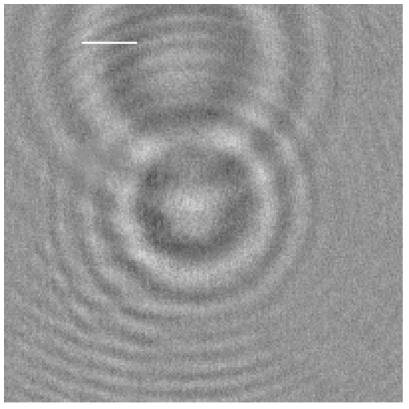

(a)

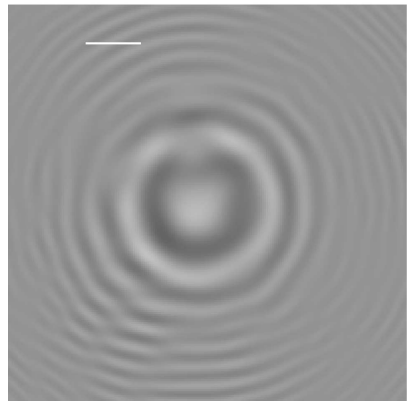

(c)

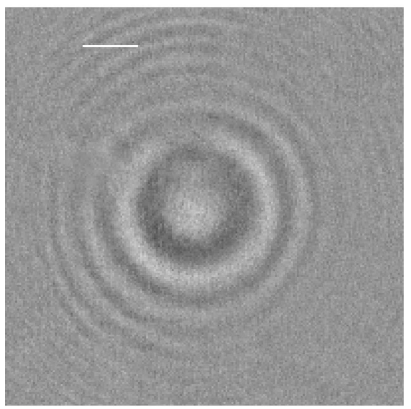

(e)

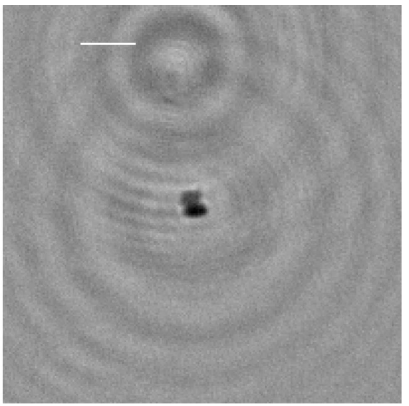

(b)

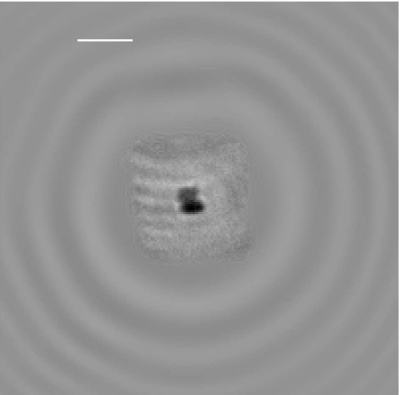

(d)

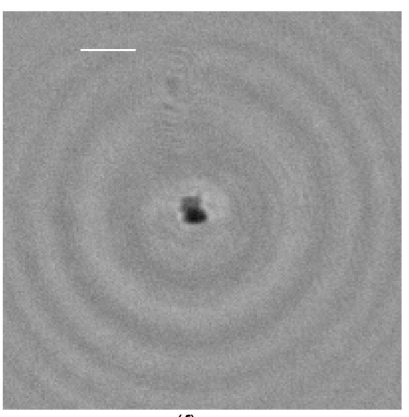

(f)

Fig. 8. To find the correct hologram of a particular object (scenedesmus alga) without any systematic error (c) we can subtract all the segmented subholograms of the nearby objects from the original measured hologram (a). Diffractions of other objects can bias the reconstruction of the actual object ((b) and (d)), if we use the original (a) or the segmented hologram (c), respectively. Conversely, if we apply the corrected hologram segmentation results (e), these biases are also eliminated (f). Here only zoomed part of the holograms and reconstructions are shown, but the algorithm uses the original, high resolution holograms (scale bars denote $20 \mu \mathrm{m}$ ). HS10000F8.eps. 